\title{
New Avirulence Genes in the Phytopathogenic Fungus Leptosphaeria maculans
}

\author{
M. H. Balesdent, A. Attard, M. L. Kühn, and T. Rouxel
}

Pathologie Végétale, INRA, Route de Saint Cyr, 78026 Versailles Cedex, France.

Accepted for publication 31 May 2002.

\begin{abstract}
Balesdent, M. H., Attard, A., Kühn, M. L., and Rouxel, T. 2002. New avirulence genes in the phytopathogenic fungus Leptosphaeria maculans. Phytopathology 92:1122-1133.

Leptosphaeria maculans, the causal agent of stem canker of oilseed rape (Brassica napus), develops gene-for-gene interactions with oilseed rape, and four L. maculans avirulence (AVR) genes (AvrLm1, AvrLm2, AvrLm4, and alml) were previously genetically characterized. Based on the analysis of progeny of numerous in vitro crosses between $L$. maculans isolates showing either already characterized or new differential interactions, this work aims to provide an overview of the AVR genes

corresponding to a complete resistance to European races of L. maculans. In both cases, a single gene control of avirulence was established (genes AvrLm3 and AvrLm7). Similarly, a single gene control of avirulence toward a $B$. rapa genotype, also resistant to European $L$. maculans isolates, was demonstrated (gene AvrLm8). Finally, a digenic control of avirulence toward B. juncea was established (genes AvrLm5 and AvrLm6). Linkage analyses demonstrated that at least four unlinked $L$. maculans genomic regions, including at least one AVR gene cluster (AvrLm1AvrLm2-AvrLm6), are involved in host specificity. The AvrLm3-AvrLm4AvrLm7 region may correspond either to a second AVR gene cluster or to a multiallelic AVR gene.
\end{abstract} that may specify incompatibility toward $B$. napus and the related species $B$. juncea and $B$. rapa. Two novel differential interactions were thus identified between L. maculans and B. napus genotypes, one of them
Additional keywords: blackleg disease, Phoma lingam.
Dissecting the genetic control of specific interactions of both the pathogen and the host plant is a prerequisite for either developing molecular characterization of the genes involved in the interaction or for developing integrated and durable management strategies to control the disease. In the case of the ascomycete Leptosphaeria maculans (anamorph Phoma lingam), the causal agent of stem canker of oilseed rape (Brassica napus L. var. oleifera) and of dry rot of swede (B. napus var. napobrassica), genetic approaches toward the identification of both avirulence (AVR) genes in the pathogen and resistance (R) genes in the host plant have been developed only very recently. Following the description of a standardized cotyledon inoculation test (36), an initial B. napus differential set was described, comprising cvs. Westar (susceptible check, spring type) and Quinta and Glacier (winter types) (22). Using this differential set, L. maculans isolates were classified into three pathogenicity groups (PG), i.e., PG2 (avirulent on cvs. Quinta and Glacier), PG3 (avirulent on cv. Quinta but virulent on cv. Glacier), and PG4 (virulent on all three cultivars). Badawy et al. (6) replaced cv. Westar by winter type $B$. napus cv. Lirabon and added Jet Neuf as a differential, leading to the description of six PG that were termed A1 to A6 $(6,23)$. PG4 isolates were thus further divided into A1 (virulent on Jet Neuf) and A5 (avirulent on Jet Neuf); PG3 into A2 (virulent on Jet Neuf) and A6 (avirulent on Jet Neuf); and PG2 into A4 and A3 (virulent and avirulent on Jet Neuf, respectively). The genetic control of these specific interactions was elucidated following genetic analyses performed both on the plant and the pathogen: (i) avirulence of PG3-A2 isolates on cv. Quinta was governed by the AVR/R gene pair AvrLml/Rlm1 (1,2); (ii) the AVR gene AvrLm2, identified in

Corresponding author: T. Rouxel; E-mail address: rouxel@versailles.inra.fr

Publication no. P-2002-0819-02R

(c) 2002 The American Phytopathological Society the PG2 isolate PHW1245, was found responsible for the induction of the hypersensitive response of cv. Glacier in which the corresponding $\mathrm{R}$ gene, $R \operatorname{lm} 2$, was genetically identified (2). The AvrLm2 locus was very closely linked to AvrLm1, also present in isolate PHW1245 (2); and (iii) incompatibility of A5 isolates toward Jet Neuf was governed by the interaction between the AVR gene AvrLm4, unlinked to AvrLm1, and the R gene Rlm4 (7). Consequently, the specific interactions described using the Kuswinanti et al. (23) differential set could be clearly explained by gene-forgene interactions, and A1 to A6 isolates could thus be considered as six races of the pathogen, differing in their AVR genes pattern. Independently, the PG2-B. napus cv. Major interaction was genetically characterized on both the plant and the pathogen: a single major locus (LEM 1) was identified in cv. Major (16) and the corresponding AVR gene, identified in the PG2 isolate PHW1245, was termed alml (29).

Many additional specific interactions were described between L. maculans and B. napus but none of them were genetically analyzed. Specific interactions were described between $B$. napus cultivars or lines Chisaya, Rafal, RX3, and Australian L. maculans isolates (9). Kutcher et al. (24) described additional interactions using B. napus cvs. Karat, Global, Marnoo, and Regent, and the two breeding lines R83-14.DH47 and R83-14.DH26. Similarly, Kuswinanti et al. (23) subdivided European PG3 and PG4 isolates into seven subgroups following cotyledon inoculations on eight B. napus differentials (Lirabon, Quinta, Glacier, Jet Neuf, Doublol, Karat, R83-14.DH47, and R83-14.DH26). Using as differentials B. napus cultivars or lines (Westar, Glacier, Quinta, Quantum, Sentry, Sprint, Val-1, and Dac-1), L. maculans PG2 isolates were subdivided into 15 distinct PG and PG3 and PG4 were subdivided into eight PG (20). The Swede cv. Tina was first described as a new source of resistance to $L$. maculans (25), but $L$. maculans isolates virulent on cv. Tina were described later (19). All these interactions are probably due to genetically uncharacterized AVR/R gene pairs. 
Finally, specific interactions between $L$. maculans and its host plants can probably be expanded to the related Brassica species $B$. rapa, B. oleracea, $B$. juncea, and $B$. nigra. Different plant genotypes within B. rapa (6,22-24), B. oleracea (6), or B. juncea (21, $23,24)$ displayed differential reactions when challenged by different L. maculans isolates. Only one of these potential specific interactions was genetically analyzed in the pathogen, following in vitro crosses involving the $B$. juncea virulent isolate IBCN18 (or M1) (11,14). This interaction was found to be inherited as a single gene in the pathogen $(11,14)$. Although clear evidence of hypersensitive response was reported in $B$. juncea when inoculated with the avirulent parent of the cross (10), this still unnamed locus was considered a virulence locus due to the lack of $B$. juncea genotypes displaying resistance toward IBCN18 (14).

Based on the analysis of progeny of numerous in vitro crosses between $L$. maculans isolates showing either already characterized or new differential interactions, the present study aims to provide an overview of the AVR genes that may specify cultivar and species-specific incompatibility toward B. napus, B. juncea, and B. rapa. Apart from identifying new L. maculans AVR genes, the present study suggests that cultivar and species-specific incompatibility may be of a similar genetic nature in this pathosystem and demonstrates that at least four unlinked L. maculans genomic regions, including one AVR gene cluster, are involved in host specificity.

\section{MATERIALS AND METHODS}

Fungal strains and culture maintenance. Isolates available at the beginning of this study, used as reference or for in vitro crosses, are listed in Table 1. The genotypes of isolates H5, 11.26.14, v11.5.1, v11.1.2, v23.1.2, v23.1.3, v23.1.11, and v23.2.1 at the three AVR loci AvrLm1, AvrLm2, and AvrLm4 were previously determined following inoculations on B. napus cultivars or lines QuintaV (Rlm1-Rlm4), Columbus (Rlml), Glacier (Rlm2), and Jet Neuf (Rlm4) (Table 1) (1,7). Isolates IBCN18, IBCN74, IBCN78, and IBCN79 belong to the International Blackleg of Crucifers Network (IBCN) collection $(32,34)$. Isolate IBCN74 (PHW1245), analyzed in a previous study (crosses 7 and 14), possessed the two AVR alleles AvrLm1 and AvrLm2 (2). Isolate Nz-T, virulent on $B$. napus var. napobrassica $\mathrm{cv}$. Tina, was further purified following single-conidia isolation as described previously (17). Eight single-conidia cultures were selected and their viru- lence on cv. Tina was controlled. Isolate Nz-T4 was selected for genetic studies. The two European PG4 isolates (i.e., possessing virulent alleles at all three AVR loci AvrLm1, AvrLm2, and AvrLm4) (7), namely UK1 from the United Kingdom and PT1 from Portugal, belong to the IMASCORE collection of European isolates $(4,34)$. All fungal cultures were maintained and stored for long term as already reported $(1,7)$.

In vitro crossing. Nine new crosses were performed for the present study (Table 2). In vitro crosses and tetrad or random ascospore progeny recovery were performed as previously established $(8,18)$. All progeny from in vitro crosses were named as described by Balesdent et al. (7). Twin isolates within each tetrad were identified by their identical electrokaryotypes $(7,26)$. Tetrads were considered complete when at least one isolate of each of the four genotypes of the tetrad was recovered. Progeny from crosses 7 and 14, which were obtained previously (2), were further characterized on B. napus cv. Jet Neuf.

Plant material. Seeds of B. napus cvs. Westar and Glacier were provided by G. Séguin-Swartz (AAF, Saskatoon, Canada) and H. K. Love (Svalof Weibull Seed, Lindsay, Canada), respectively. Seeds of cvs. Symbol, Vivol, Capitol, Doublol, Bristol, and Columbus were obtained from Semences Cargill (Boissay, France). The Quinta S2-V95 line, possessing both Rlm1 and Rlm4 (7), was further multiplied by self-pollination of plants that were first checked for the presence of the two R genes following inoculation with reference isolates. Self-pollination took place in the greenhouse under pollen-proof bags to avoid pollen contamination. The resulting S3 line was termed QuintaV.

Seeds of B. napus cvs. Jet Neuf, Express, Falcon, and Darmor and $B$. juncea cvs. Aurea and Picra were obtained from M. Renard (INRA, Amélioration des Plantes, Le Rheu, France) and further multiplied by self-pollination. The B. napus line Falcon-MX, also provided by $M$. Renard, is a $B$. napus $-B$. juncea recombinant line containing the Jlm1 resistance gene from B. juncea (12; A. M. Chèvre, personal communication).

Two individual $B$. juncea cv. Aurea plants were selected for their unusual behavior when inoculated with some progeny isolates from cross 22 and were self-pollinated. From the two S1 resulting lines, approximately 10 plants per line were selected following inoculation with the same isolates. Only plants showing the same interaction phenotype as the original plant were chosen for subsequent mass-pollination in the greenhouse under pollenproof bags, resulting in S2 lines 150-2-1 and 151-2-1.

TABLE 1. List and characteristics of field or laboratory isolates used in this study

\begin{tabular}{|c|c|c|c|c|c|c|c|}
\hline Isolate number & $\begin{array}{l}\text { Country } \\
\text { of origin }\end{array}$ & Origin & Source & Other name & Mating type & $\begin{array}{l}\text { Available data on pathogenicity } \\
\text { or avirulence (AVR) allele }\end{array}$ & Reference \\
\hline H5 & France & $\mathrm{F}^{\mathrm{b}}$ & C. Gall & & - & avrLm1-avrLm2-avrLm4 & $(1,7)$ \\
\hline IBCN18 & Australia & $\mathrm{F}$ & B. Howlett & M1 & - & Virulent on Brassica juncea & $(11,34)$ \\
\hline IBCN78 & Australia & $\mathrm{F}$ & P. H. Williams & PHW1321/WA43 & + & $\mathrm{PG} 2^{\mathrm{c}}$ & (34) \\
\hline IBCN79 & Australia & $\mathrm{F}$ & P. H. Williams & PHW1325/WA52 & + & PG2 & (34) \\
\hline IBCN74 & France & $\mathrm{F}$ & P. H. Williams & PHW1245 & + & $\begin{array}{c}\text { AvrLm1-AvrLm2 } \\
\text { alm1 }\end{array}$ & $\begin{array}{l}(2) \\
(29)\end{array}$ \\
\hline $\mathrm{Nz}-\mathrm{T}$ & New Zealand & $\mathrm{F}$ & S. Gowers & $\mathrm{T}$ & + & $\begin{array}{c}\text { Avirulent on Jet Neuf } \\
\text { Virulent on B. napus cv. Tina }\end{array}$ & $\begin{array}{c}\text { (6; this study) } \\
\text { (19) }\end{array}$ \\
\hline 11.26 .14 & France & $\mathrm{L}$ & Cross $11^{\mathrm{d}}$ & $\ldots$ & + & avrLm1-avrLm2-avrLm4 & $(1,7)$ \\
\hline v11.1.5 & France & $\mathrm{L}$ & Cross 11 & $\ldots$ & - & AvrLm1-avrLm2-avrLm4 & $(7)$ \\
\hline v11.1.2 & France & $\mathrm{L}$ & Cross 11 & $\ldots$ & + & AvrLm1-avrLm2-avrLm4 & (7) \\
\hline v23.2.1 & France & $\mathrm{L}$ & Cross 23 & $\ldots$ & + & avrLm1-avrLm2-AvrLm4 & (7) \\
\hline $\mathrm{v} 23.1 .2$ & France & $\mathrm{L}$ & Cross 23 & $\mathrm{JN} 2$ & - & avrLm1-avrLm2-avrLm4 & (7) \\
\hline $\mathrm{v} 23.1 .3$ & France & $\mathrm{L}$ & Cross 23 & JN3 & + & AvrLm1-avrLm2-AvrLm4 & (7) \\
\hline v23.1.11 & France & $\mathrm{L}$ & Cross 23 & JN11 & - & AvrLm1-avrLm2-AvrLm4 & (7) \\
\hline UK1 & England & $\mathrm{F}$ & B. Fitt & 95 A6 & + & PG4-A1 & (34) \\
\hline PT1 & Portugal & $\mathrm{F}$ & J. S. Dias & $\ldots$ & $\mathrm{nd}^{\mathrm{e}}$ & PG4-A1 & (34) \\
\hline
\end{tabular}

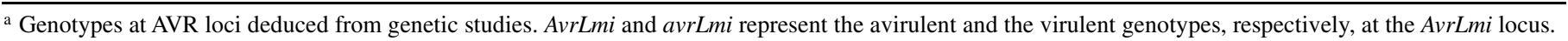

${ }^{\mathrm{b}} \mathrm{F}$, field single-ascospore isolate; L, laboratory isolate originating from in vitro crosses.

c PG, pathogenicity groups according to the terminology of Koch et al. (22).

${ }^{\mathrm{d}}$ Crosses 11 and 23 are described in Balesdent et al. (7).

e nd, not determined. 
Six B. napus-derived lines and one B. rapa-derived line (156-2-1) were obtained following a preliminary screening of gene bank genotypes for resistance to isolates v11.1.1, v11.1.2, and v23.2.1 (T. Rouxel, E. Willner, and M. H. Balesdent, unpublished data) (Table 3). In all cases, only a few plants resistant to the three isolates were identified among the screened accessions. Depending on the lines, the selected resistant plants were either self pollinated individually (lines 149-2-1, 05-1-1, 04-1-2, 156-2-1, 23-1-1, and 22-1-1) or mass pollinated (line 148-1-1). When the S1 populations showed a heterogeneous behavior after inoculation with reference isolates (lines 05-1-1, 149-2-1, 23-1-1, 22-1-1, and 1562-1) a second round of mass pollination took place with selected $\mathrm{S} 1$ plants (=S2 generation). In one case however, the $\mathrm{S} 2$ resulting line was still heterogeneous when inoculated with reference isolates v11.1.1, v11.1.2, and Nz-T4 with two main phenotypic classes, the first being resistant to v11.1.1 and v11.1.2 but susceptible to $\mathrm{Nz}-\mathrm{T} 4$, the second being susceptible to the three isolates. Five plants of each phenotypic class were selected and mass pollinated. The two resulting S3 lines, 23-1-1 and 22-1-1, respectively, were $100 \%$ homogeneous and similar to the parental phenotypes. Lines 04-1-2, 22-1-1, and 23-1-1 were derived from B. napus var. oleifera cultivars, whereas lines 149-2-1, 148-1-1, and 05-1-1 were derived from $B$. napus var. napobrassica.
Pathogenicity tests. Isolates were inoculated on cotyledons of the brassica genotypes as previously described $(1,7)$. Plants were incubated in a growth chamber at $16 / 24^{\circ} \mathrm{C}$ (night/day) with a $12 \mathrm{~h}$ photoperiod. Symptoms were scored 14 to 27 days after inoculation using the IMASCORE rating scale comprised of six infection classes (IC), in which IC1 to IC3 are resistance responses and IC4 to IC6 are susceptibility symptoms (7). Each isolate was inoculated on at least 10 different plants, and experiments were repeated twice. Finally, the result of each isolate-line interaction was recorded as compatible (more than $80 \%$ of susceptibility responses, the isolate is virulent [V]) or incompatible (more than $80 \%$ of resistance responses, the isolate is avirulent [A]). In a few cases, the isolate-line interaction was heterogeneous, with a majority of resistance responses (IC1 to IC3) but with a significant (more than 20\%) percentage of susceptibility symptoms (IC4 to IC6), probably due to plant genotype heterogeneity (Fig. 1).

Genetic analyses. For each interaction, the ratio of $\mathrm{A} / \mathrm{V}$ progeny was tested for deviation from Mendelian expectation by chisquare analysis. For random progeny in which more than two AVR genes segregated, linkage analyses among AVR loci was performed using the Mapmaker/EXP 3.0 software (available online; Whitehead/MIT Center for Genome Research, Cambridge,

TABLE 2. List of the in vitro crosses described in this study

\begin{tabular}{|c|c|c|c|c|}
\hline \multirow[b]{2}{*}{ Cross } & \multicolumn{2}{|c|}{ Parental isolates $^{\mathrm{a}}$} & \multicolumn{2}{|c|}{ Progeny } \\
\hline & 1 & 2 & Recovered & Selected for subsequent crosses \\
\hline 19 & IBCN79 & H5 & 1 complete +6 incomplete tetrads & $\ldots$ \\
\hline 20 & IBCN18 & 11.26 .14 & 13 ascospores from 5 asci & 20.3 .04 (Mat-) \\
\hline 22 & 20.3 .04 & 11.26 .14 & 10 complete tetrads & 22.2.02 (Mat-); 22.2 .03 (Mat+) \\
\hline 24 & 22.2 .03 & $\mathrm{v} 11.1 .5$ & 70 random ascospores & $\ldots$ \\
\hline 29 & 22.2 .02 & 22.2 .03 & 67 random ascospores & v29.3.1 (Mat-) \\
\hline 34 & v29.3.1 & $\mathrm{v} 23.1 .3$ & 62 random ascospores & $\ldots$ \\
\hline 30 & Nz-T4 & $\mathrm{v} 23.1 .2$ & 66 random ascospores & $\ldots$ \\
\hline 33 & Nz-T4 & $\mathrm{v} 23.1 .11$ & 48 random ascospores & $\ldots$ \\
\hline 35 & IBCN78 & $\mathrm{v} 23.1 .11$ & 43 random ascospores & $\ldots$ \\
\hline
\end{tabular}

a Additional information on the isolates is provided in Table 1.

TABLE 3. Interaction phenotypes between selected Brassica cultivars or lines and Leptosphaeria maculans isolates used as reference or for genetic analyses

\begin{tabular}{|c|c|c|c|c|c|c|c|c|c|c|c|c|c|c|}
\hline & \multicolumn{14}{|c|}{ Plant genotypes and known or putative resistance genes } \\
\hline & \multirow{2}{*}{\multicolumn{7}{|c|}{ Reference cultivars }} & \multicolumn{7}{|c|}{ Additional lines displaying new specific interactions } \\
\hline & & & & & & & & \multirow{2}{*}{\multicolumn{2}{|c|}{$\begin{array}{l}148-1-1 \\
05-1-1 \\
04-1-2 \\
23-1-1 \\
(R \operatorname{lm} 7)\end{array}$}} & \multirow[b]{2}{*}{$\begin{array}{l}22-1-1 \\
(R \operatorname{lm} 3)\end{array}$} & \multirow{2}{*}{$\begin{array}{c}\text { B. juncea } \\
\text { cvs. Picra } \\
\text { and Aurea } \\
(R \operatorname{lm} 5) \\
(R \operatorname{lm} 6)\end{array}$} & \multirow{2}{*}{$\begin{array}{c}\text { B. juncea } \\
\text { lines 150-2-1 } \\
\text { and 151-2-1 } \\
\quad(\text { Rlm5 })\end{array}$} & \multirow{2}{*}{$\begin{array}{c}\text { Falcon-MX } \\
J \operatorname{lm} 1 \\
(R \operatorname{lm} 4) \\
(R \operatorname{lm} 6=J \operatorname{lm} 1)\end{array}$} & \multirow{2}{*}{$\begin{array}{c}156-2-1 \\
(R \operatorname{lm} 8)\end{array}$} \\
\hline & Westar & $\begin{array}{c}\text { QuintaV } \\
\text { Rlm1 } \\
\text { Rlm4 }\end{array}$ & $\begin{array}{c}\text { Columbus } \\
\text { Rlm1 } \\
(R \operatorname{lm} 3)^{\mathrm{a}}\end{array}$ & $\begin{array}{c}\text { Glacier } \\
\text { Rlm2 } \\
(\text { Rlm3) }\end{array}$ & $\begin{array}{l}\text { Bristol } \\
(R \operatorname{lm} 2)\end{array}$ & $\begin{array}{c}\text { Jet } \\
\text { Neuf } \\
\text { Rlm4 }\end{array}$ & $\begin{array}{l}\text { Falcon } \\
(\text { Rlm4) }\end{array}$ & & & & & & & \\
\hline $\begin{array}{l}\text { PHW1245 } \\
\text { AvrLm1 AvrLm2 AvrLm4 }\end{array}$ & $\mathrm{S}^{\mathrm{c}}$ & $\mathrm{R}$ & $\mathrm{R}$ & $\mathrm{R}$ & $\mathrm{R}$ & $\mathrm{R}$ & $\mathrm{R}$ & $\mathrm{R}$ & $\mathrm{R}$ & $\mathrm{S}$ & $\mathrm{R}$ & $\mathrm{R}$ & $\mathrm{R}$ & $\mathrm{R}$ \\
\hline $\begin{array}{l}\mathrm{H} 5, \mathrm{UK} 1, \mathrm{PT} 1, \mathrm{v} 23.1 .2 \\
\text { avrLm1 avrLm2 avrLm4 }\end{array}$ & $\mathrm{S}$ & $\mathrm{S}$ & $\mathrm{S}$ & $\mathrm{S}$ & $\mathrm{S}$ & $\mathrm{S}$ & $\mathrm{S}$ & $\mathrm{R}$ & $\mathrm{R}$ & $\mathrm{S}$ & $\mathrm{R}$ & $\mathrm{R}$ & $\mathrm{R}$ & $\mathrm{R}$ \\
\hline $\begin{array}{l}\text { v23.1.3, v23.1.11 } \\
\text { AvrLm1 avrLm2 AvrLm4 }\end{array}$ & $\mathrm{S}$ & $\mathrm{R}$ & $\mathrm{R}$ & $\mathrm{S}$ & $\mathrm{S}$ & $\mathrm{R}$ & $\mathrm{R}$ & $\mathrm{R}$ & $\mathrm{R}$ & $\mathrm{S}$ & $\mathrm{R}$ & $\mathrm{R}$ & $\mathrm{R}$ & $\mathrm{R}$ \\
\hline $\begin{array}{l}\text { v11.1.2 } \\
\text { AvrLm1 avrLm2 avrLm4 }\end{array}$ & $S$ & $\mathrm{R}$ & $\mathrm{R}$ & $S$ & $S$ & $\mathrm{~S}$ & $S$ & $\mathrm{R}$ & $\mathrm{R}$ & $\mathrm{S}$ & $\mathrm{R}$ & $\mathrm{R}$ & $\mathrm{R}$ & $\mathrm{R}$ \\
\hline $\begin{array}{l}\text { v23.2.1 } \\
\text { avrLm1 avrLm2 AvrLm4 }\end{array}$ & $\mathrm{S}$ & $\mathrm{R}$ & $\mathrm{S}$ & $\mathrm{S}$ & $\mathrm{S}$ & $\mathrm{R}$ & $\mathrm{R}$ & $\mathrm{R}$ & $\mathrm{R}$ & $\mathrm{S}$ & $\mathrm{R}$ & $\mathrm{R}$ & $\mathrm{R}$ & $\mathrm{R}$ \\
\hline $\begin{array}{l}\text { IBCN18 }^{\mathrm{d}} \\
\text { AvrLm1 AvrLm2 AvrLm4 }\end{array}$ & $\mathrm{S}$ & $\mathrm{R}$ & $\mathrm{R}$ & $\mathrm{R}$ & $\mathrm{R}$ & $\mathrm{R}$ & $\mathrm{R}$ & $\mathrm{R}$ & $\mathrm{R}$ & $S$ & $S$ & $S$ & $\mathrm{R}$ & $S$ \\
\hline $\begin{array}{l}\text { Nz-T4 } \\
\text { avrLm1 avrLm2 avrLm4 }\end{array}$ & $\mathrm{S}$ & $\mathrm{S}$ & $\mathrm{S}$ & $\mathrm{S}$ & $\mathrm{S}$ & $\mathrm{S}$ & $\mathrm{S}$ & $\mathrm{S}$ & $\mathrm{S}$ & $\mathrm{S}$ & $\mathrm{R}$ & $\mathrm{R}$ & $\mathrm{R}$ & $\mathrm{R}$ \\
\hline $\begin{array}{l}\text { IBCN78, IBCN79 } \\
\text { AvrLm1 avrLm2 avrLm4 }\end{array}$ & $\mathrm{S}$ & $\mathrm{R}$ & $\mathrm{R}$ & $\mathrm{R}$ & $\mathrm{S}$ & $S$ & $\mathrm{~S}$ & $\mathrm{R}$ & $S$ & $\mathrm{R}$ & $\mathrm{R}$ & $\mathrm{R}$ & $\mathrm{R}$ & $\mathrm{R}$ \\
\hline
\end{tabular}

${ }^{a}$ Resistance genes between brackets are putative resistance genes deduced from the present study.

${ }^{\mathrm{b}} A v r L m i$, the isolate is avirulent, avrLmi, the isolate is virulent; the genotypes are given for the three avirulent (AVR) alleles AvrLm1, AvrLm2, and AvrLm4.

${ }^{\mathrm{c}} \mathrm{R}$, the line is resistant (incompatible interaction); $\mathrm{S}$, the line is susceptible (compatible interaction).

${ }^{\mathrm{d}}$ For isolates IBCN18, Nz-T4, IBCN78, and IBCN79, the AVR alleles at AvrLm1, AvrLm2, and AvrLm4 loci are deduced from the present study. 
MA) with a LOD score of 4.0 and a maximum recombination frequency of 20 centimorgans $(\mathrm{cM})$.

\section{RESULTS}

AVR genes in the reference isolates and $R$ genes in the reference cultivars. From previous genetic analyses, the genotypes of reference isolates were identified at the three loci AvrLml, AvrLm2, and AvrLm4 (Table 3) (7). PHW1245, previously described as possessing AvrLm1 and AvrLm2 (2), was avirulent on Jet Neuf (6) (Table 3). In order to better characterize the AVR genes present in PHW1245, the entire progeny of crosses 7 (six tetrads) and 14 (two tetrads) were further tested for virulence on Jet Neuf. The avirulence of PHW1245 on Jet Neuf segregated 1:1 in all tetrads from crosses 7 and 14. This result suggested that PHW1245 also possesses AvrLm4, recognizing Rlm4 present in Jet Neuf and QuintaV (Table 3). In addition, three parental ditypes (PD), four nonparental ditypes, and one tetratype (TT) were observed between AvrLm4 and AvrLml (i.e., segregation of avirulence on Rlml harboring cvs. Vivol, Doubol, Capitol, and Columbus [7]) in the eight tetrads from crosses 7 and 14, confirming the genetic independence between AvrLml and AvrLm4 (7).

Following inoculation of cv. Falcon with reference isolates, it was concluded that Falcon possesses $R \operatorname{lm} 4$, because it was resistant to all three isolates possessing AvrLm4 (Table 3). Line 22-1-1 was considered as possessing none of the $\mathrm{R}$ genes $R \operatorname{lm} 1, R \operatorname{lm} 2$, or $R \operatorname{lm} 4$ because it was susceptible to all reference isolates. Finally, because Bristol behaved similarly to Glacier, it was hypothesized that Bristol possesses Rlm2 (Table 3). All other B. napus, B. rapa, and $B$. juncea cultivars or lines were resistant to all reference isolates, including the three European PG4 field isolates H5, UK1, and PT1 (Table 3).

Evidence for new specific interactions. Isolates IBCN18, IBCN78, IBCN79, and Nz-T4 presented novel interaction phenotype patterns compared with those involving the AvrLm1/Rlml, AvrLm2/Rlm2, or AvrLm4/Rlm4 gene pairs (Table 3). In accordance with Chen et al. (11), IBCN18 was virulent on B. juncea cvs. Picra and Aurea, as well as on the two B. juncea-derived lines 150-2-1 and 151-2-1. However, in the present study, this isolate was avirulent on Falcon-MX (Table 3). IBCN18 was also virulent on the B. rapa-derived line 156-2-1, whereas it was avirulent on all cultivars, except cv. Westar, from the differential set of Kuswinanti et al. (23). This suggested that isolate IBCN18 may possess AVR alleles at all three AVR loci AvrLm1, AvrLm2, and AvrLm4.

In contrast to all above-mentioned isolates, IBCN78, IBCN79, and Nz-T4 were virulent on the lines 148-1-1, 05-1-1, 04-1-2, and 23-1-1, which were independently selected for their resistance to European PG4 isolates. Finally, isolates IBCN78 and IBCN79, but not Nz-T4, were avirulent on Glacier (but not on Bristol), QuintaV, Columbus, and on lines 22-1-1 and 149-2-1 (Table 3). Although the QuintaV-IBCN78 or IBCN79 interactions could be simply attributed to the occurrence of AvrLml in these isolates, all other interactions presented above suggested the occurrence of new gene-for-gene interactions.

AvrLm1, AvrLm2, and AvrLm4 alleles are present in isolate IBCN18. IBCN18 was crossed with the PG4 isolate 11.26.14 (cross 20; Tables 2 and 4). Very few progeny isolates, and no complete tetrads, could be recovered from this cross (Table 4). However, it was evident after analysis of this progeny that all differential interactions expressed by the parental isolates of cross 20 segregated in the progeny, because seven distinct phenotypic classes were recovered in the progeny (Table 4).

Cosegregation of avirulence on Columbus, QuintaV, Doublol, Bristol, and Glacier could be explained by the hypothesis that isolate IBCN18 possessed the two tightly linked AVR alleles AvrLmI and AvrLm2, whereas the independent segregation of avirulence on Rlm4 cvs. Jet Neuf and Falcon is consistent with the hypothe- sis that AVR allele AvrLm4 is present in isolate IBCN18 (Table 4). As expected by the occurrence of both $R \operatorname{lm} 1$ and $R \operatorname{lm} 4$ in QuintaV, all isolates in the progeny that were avirulent either on Columbus or on Jet Neuf were also avirulent on QuintaV (Table 4).

The whole progeny remained avirulent on lines 04-1-2 and 05-1-1, as were the two parents of the cross (data not shown). In contrast, 6 of 13 progeny isolates were virulent on Falcon-MX, although the two parent isolates induced a resistance reaction on this line (Table 4). This could suggest that two independent AVR genes, recognizing two $\mathrm{R}$ genes with epistatic effects present in Falcon-MX, are segregating in the progeny. It has to be noticed that all isolates that were avirulent on Jet Neuf and Falcon (i.e., possessing the AvrLm4 allele) were avirulent on Falcon-MX. This indicated that $R \operatorname{lm} 4$, present in cv. Falcon, was also present in the derived line Falcon-MX and could be one of the two Falcon-MX $\mathrm{R}$ genes. Due to the small number of progeny analyzed, the segregation of avirulence on the two B. juncea cvs. Picra and Aurea fits

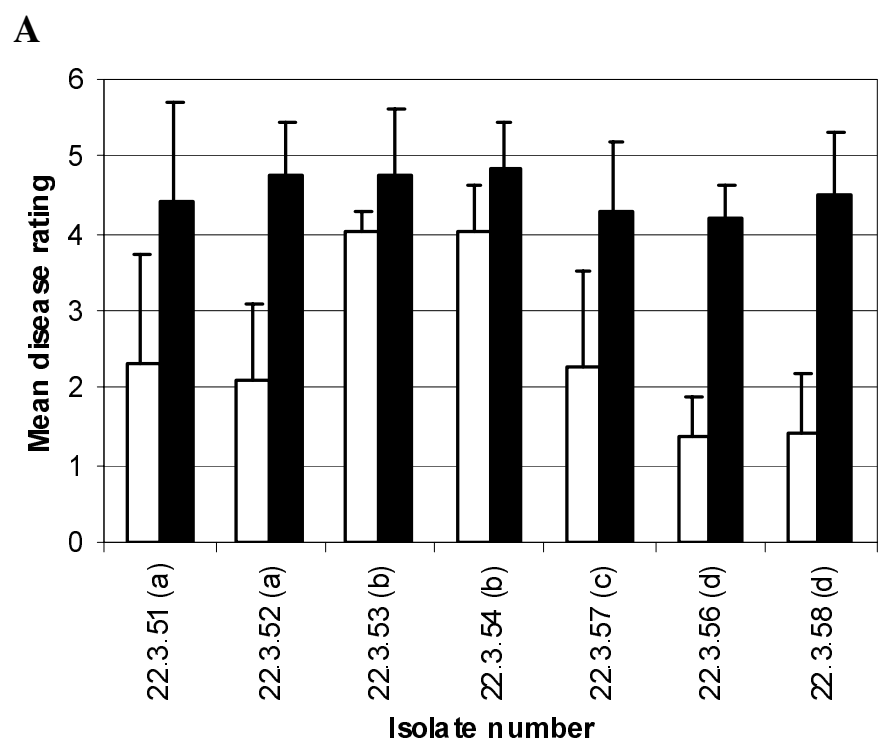

B

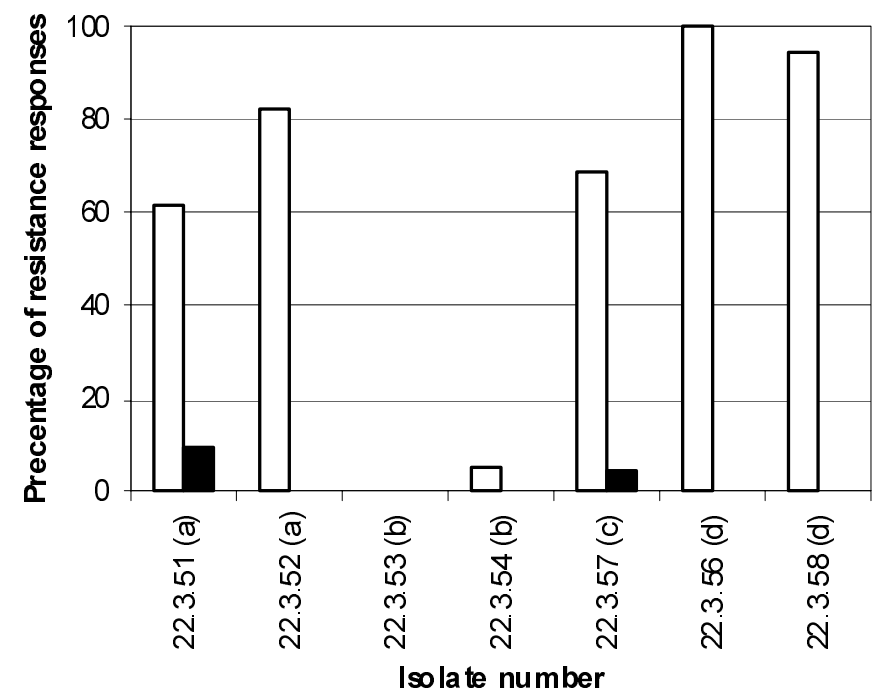

Fig. 1. An example of avirulence segregation of Leptosphaeria maculans on Brassica juncea cv. Picra in one complete tetrad (seven isolates, four genotypes) from cross 22. Symptoms were rated 15 days after inoculation using six infection classes (IC1 to IC6) for a rating scale (7). A, Mean disease rating (the vertical line is the standard deviation) and $\mathbf{B}$, percentage of resistance responses (IC1 to IC3) of 16 to 23 inoculation sites for each isolate-plant genotype interaction. Isolate numbers are described in Table 5, and isolates with the same letter are twin isolates. White bars, $B$. juncea cv. Picra; and black bars, B. napus cv. Westar as a susceptible check. 
both a $50: 50 \mathrm{~A} / \mathrm{V}$ ratio (chi-square $=0.692 ; P=0.405$ ) or a $75: 25$ ratio (chi-square $=1.256 ; P=0.262$ ). Finally, virulence of IBCN18 on the two $B$. juncea-derived lines 150-2-1 and 151-2-1 and on the $B$. rapa-derived line 156-2-1 segregated in cross 20 independently from all above-mentioned interactions (Table 4).

To increase the progeny, a backcross (cross 22) was performed between isolate 20.3.04, displaying the same interaction phenotype as IBCN18, except that it was virulent on Jet Neuf and 11.26.14 (Tables 2 and 5). Cross 22 was much more fertile than cross 20, because 10 complete tetrads could be recovered. The whole progeny was virulent on Jet Neuf and Falcon (Table 5). As expected for a single gene control of avirulence on $R \operatorname{lm} 1$ and on $R \operatorname{lm} 2$ cultivars, a 2:2 A/V ratio was observed within each tetrad when inoculated on a range of cultivars possessing Rlml (Capitol, Columbus, Doublol, and Vivol) on the one hand, or on Glacier, Bristol, and the two additional cvs. Express and Symbol, also possessing Rlm2 (T. Rouxel and M. H. Balesdent, unpublished data), on the other hand (Table 5). In all cases, twin isolates displayed the same interaction phenotypes. For 9 of 10 tetrads, isolates that were avirulent on Rlml cultivars were also avirulent on Bristol, Glacier, Express, and Symbol. For one tetrad however (tetrad 3), a tetratype was observed, with one twin pair avirulent on $R \operatorname{lm} 1$ genotypes but not on Rlm2 genotypes, and one twin pair virulent on $R \operatorname{lm} 1$ genotypes but not on Rlm2 genotypes (Table 5).

To further support the hypothesis that isolate IBCN18 possessed the AvrLml AVR allele, rather than another independent AVR gene conferring an identical interaction spectrum, one additional cross (cross 24) was performed (Table 2). In this cross, isolate 22.2.03 (putatively AvrLm1-avrLm2) was crossed with isolate v11.1.5 possessing the AvrLm1 allele. From the 70 progeny isolates recovered, 56 isolates were assessed for avirulence on Columbus. All of them were avirulent and no recombinant isolate (i.e., virulent on $R \operatorname{lm} 1$ ) could be detected (Table 6).

Identification of three virulence alleles in IBCN18: avrLm5, avrLm6, and avrLm8. Crosses 22, 24, and 29 were used to analyze the genetic control of avirulence on $B$. juncea and $B$. rapa genotypes. For 9 of the 10 tetrads from cross 22, a 3:1 A/V ratio was observed on B. juncea cvs. Picra or Aurea, whereas a 2:2 A/V segregation was observed in one tetrad only (tetrad 6; Table 5). The predominantly observed $3: 1 \mathrm{~A} / \mathrm{V}$ ratio on $B$. juncea cultivars is most likely to correspond to the occurrence of two (or more) un- linked AVR genes present in isolate 11.26.14, each of them being able to induce the resistance response of $B$. juncea. For most tetrads however, the result of the interaction on $B$. juncea was not always easily scored as a compatible or incompatible interaction. One genotype within each tetrad was usually clearly virulent, another one (or two) clearly avirulent, the others were often scored as heterogeneous, mainly because of a high standard deviation of mean ratings due to heterogeneity of plant response (Fig. 1; Table 5). These heterogeneous responses were more frequently observed on cv. Aurea than on cv. Picra (data not shown). $B$. juncea lines 150-2-1 and 151-2-1 were obtained by selfing individual $B$. juncea $\mathrm{cv}$. Aurea plants that were fully susceptible to isolates revealing such a heterogeneous interaction phenotype. Interaction phenotypes of cross 22 progeny were identical on 1502-1 and 151-2-1 and segregated with a 2:2 ratio in all tetrads (Table 5). Similarly, a 2:2 A/V segregation was observed for each tetrad on Falcon-MX, and recombination between avirulence on Falcon-MX, and on the 150-2-1 and 151-2-1 lines, was very frequently observed (one PD and nine TT) (Table 5). From these data, the following hypothesis is proposed: avirulence on $B$. juncea cvs. Aurea and Picra is governed by two pairs of genes termed AvrLm5/Rlm5 (Rlm5 being present in the two B. juncea cultivars and in lines 150-2-1 and 151-2-1 but not in Falcon-MX) and AvrLm6/Rlm6 (Rlm6 being present in the two B. juncea cultivars and in Falcon-MX but not in lines 150-2-1 and 151-2-1). The genotypes of parent isolates of crosses 20 and 22 would be AvrLm5-AvrLm6 (isolate 11.26.14) and avrLm5-avrLm6 (isolates IBCN18 and 20.3.04). When one of these two AVR alleles is present, the isolate is avirulent on B. juncea, whereas virulence is only observed when the two virulent alleles are present. The characterization of cross 22 progeny on lines Falcon-MX, 150-2-1, and 151-2-1 allowed us to determine the putative genotype of each isolate at the two loci AvrLm5 and AvrLm6 (Table 5). The two clear interaction phenotypes observed within each tetrad corresponded either to the avrlm5-avrlm6 or to the AvrLm5-Avrlm6 genotypes, whereas the heterogeneous responses were observed when only one AVR allele was present (either AvrLm5 or AvrLm6) (Table 5). This heterogeneous response, attributable to plant heterogeneity, would be more frequently observed when one $\mathrm{R}$ gene is lacking than if two $\mathrm{R}$ genes are lacking in one individual plant. Our hypothesis is further supported by segregation data from cross

TABLE 4. Interaction phenotypes of parental isolates and progeny of cross 20 (IBCN18 $\times 11.26 .14$ ) on a range of Brassica napus, B. rapa, and B. juncea cultivars or lines

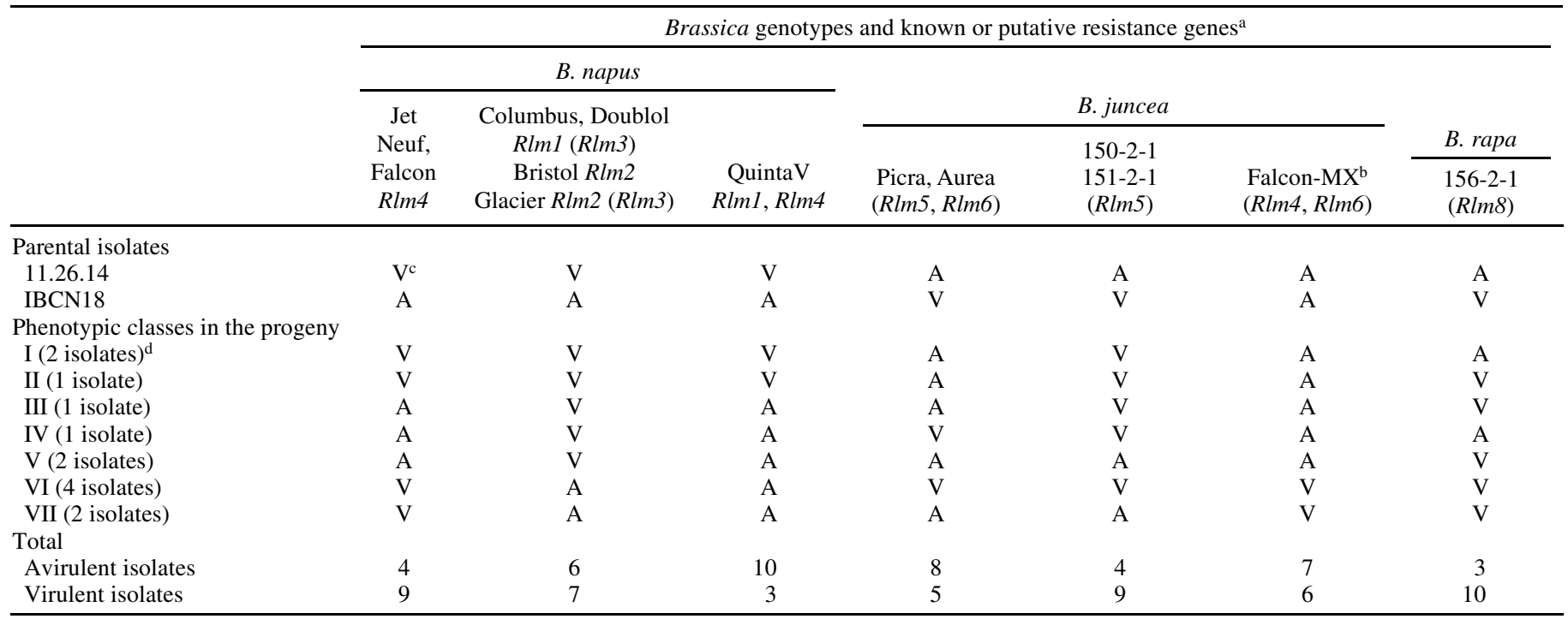

${ }^{a}$ Resistance genes between brackets are putative resistance genes deduced from the present study.

${ }^{\mathrm{b}}$ Falcon-MX is a $B$. napus recombinant line with the $B$. juncea $\mathrm{R}$ gene $\operatorname{Jlm} 1$ (12), termed $\operatorname{Rlm} 6$ in the present study.

${ }^{c} \mathrm{~A}$, the isolate is avirulent (incompatible interaction); $\mathrm{V}$, the isolate is virulent (compatible interaction).

${ }^{\mathrm{d}}$ Number between brackets is the number of recovered isolates for each phenotypic class. 
24 in which only AvrLm5 segregates (Tables 5 and 6). All tested progeny isolates from cross 24 were avirulent on $B$. juncea cvs. Picra and Aurea, whereas avirulence on lines 150-2-1 and 151-2-1 segregated as a single gene (Table 6).

Finally, a 2:2 A/V segregation was observed within each tetrad when inoculated on B. rapa line 156-2-1, suggesting again a single gene control of avirulence on this line. Because the segregation of this avirulence, in crosses 20 and 22, appeared independent from all other AVR genes previously characterized (Tables 4 and 5), it is suggested that a new AVR locus, termed AvrLm8, governs the interaction phenotype on line 156-2-1.

Linkage analyses between the AVR loci. Segregation of avirulence or virulence of progeny from crosses 20 and 22 indicated genetic independence between the AvrLm1-AvrLm2 region, AvrLm4, AvrLm5, and AvrLm8, whereas AvrLm6 cosegregated with AvrLm2. However, too few meiotic events were analyzed with these two crosses ( 5 and 10 for crosses 20 and 22, respectively) to assess genetic distance between all these loci. In cross 29 , the two sister isolates 22.2 .02 (avrLm1 AvrLm2 avrLm4 AvrLm5 avrLm6 avrLm8) and 22.2.03 (AvrLm1 avrLm2 avrLm4 avrLm5 AvrLm6 AvrLm8) were crossed (Tables 2 and 5) and AvrLm1, AvrLm2, AvrLm5, AvrLm6, and AvrLm8 were all segregating as simple traits in the resulting progeny (Table 6). When considering only AvrLm1 and AvrLm2, three phenotypic classes were recovered, i.e., the two parental phenotypes AvrLm1-avrLm2 (35 isolates) and avrLm1-AvrLm2 (31 isolates), and the recombinant phenotype

TABLE 5. Tetrad analysis of avirulence segregation of Leptosphaeria maculans progeny in cross $22(20.3 .04 \times 11.26 .14)$ on a range of Brassica napus, B. juncea, and $B$. rapa lines

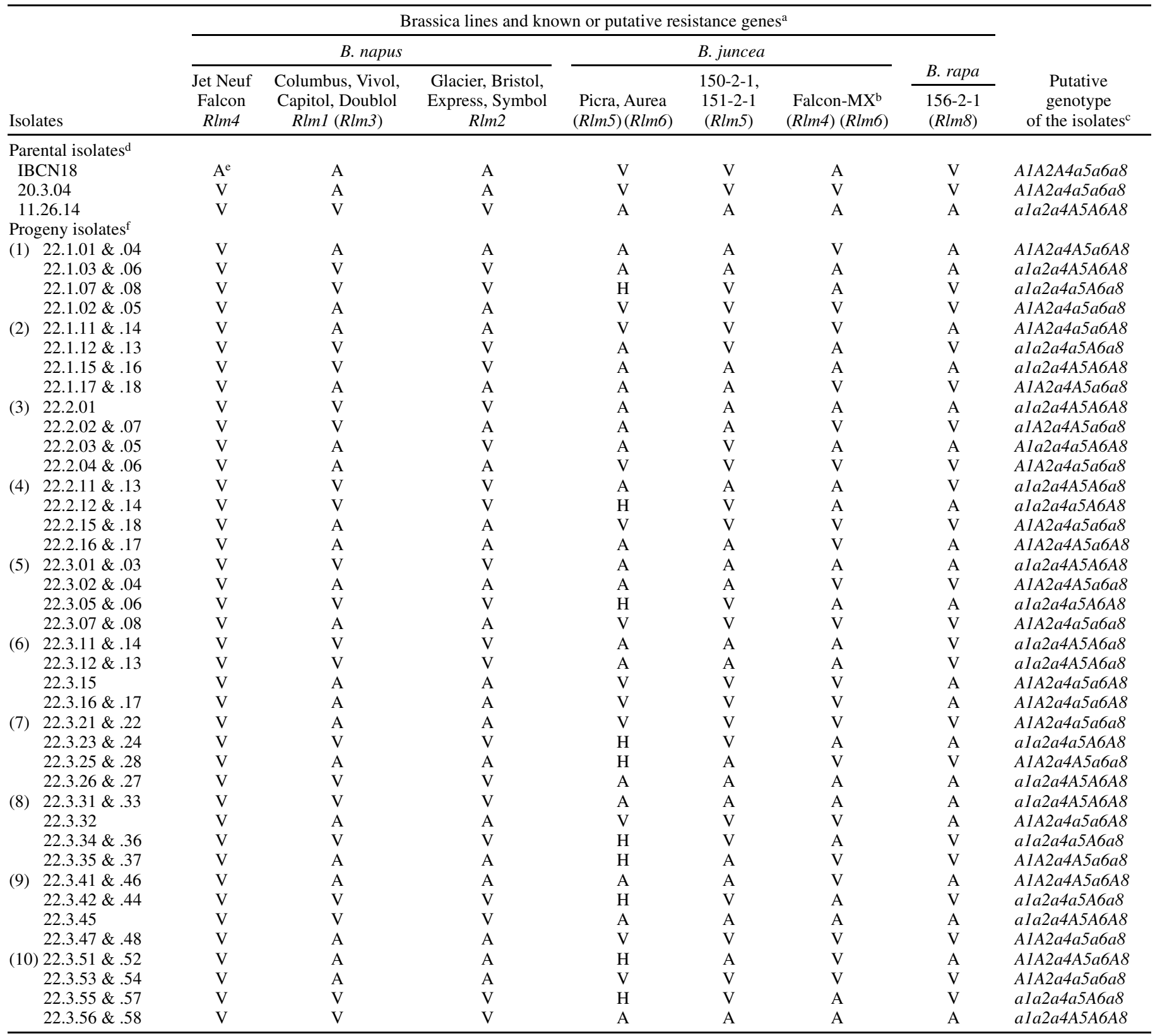

${ }^{a} \mathrm{R}$ genes between brackets are putative $\mathrm{R}$ genes deduced from the present study.

${ }^{\mathrm{b}}$ Falcon-MX is a $B$. napus recombinant line with the B. juncea $\mathrm{R}$ gene $J l m 1$ (12) termed $R \operatorname{lm} 6$ in the present study.

${ }^{\mathrm{c}} A i$, presence of the avirulent allele at the AvrLmi locus; $a i$, presence of the virulent allele at the AvrLmi locus.

${ }^{\mathrm{d}}$ Isolate 20.3.04 is a F1 progeny from cross IBCN18 × 11.26.14 (Table 2).

${ }^{\mathrm{e}} \mathrm{A}$, the isolate is avirulent; $\mathrm{V}$, the isolate is virulent; $\mathrm{H}$, the interaction phenotype is heterogeneous, with a significant (more than $20 \%$ ) percentage of susceptibility symptoms (infection class (IC)4 to IC6), but however a majority of resistance responses (IC1 to IC3).

${ }^{\mathrm{f}}$ Each tetrad is identified by its number, indicated between brackets. Isolates on the same line are twin isolates, as identified following pulsed field gel electrophoresis. 
AvrLm1-AvrLm2 (one isolate). Thus, only 1 recombinant isolate out of 67 was identified between AvrLm1 and AvrLm2 in cross 29, confirming the tight linkage $(1.5 \mathrm{cM})$ between these two loci. No recombinant isolates between AvrLm2 and AvrLm6 could be recovered in cross 29.

Cross 34 was set up to include AvrLm4 in the linkage analysis. Segregation data confirmed the single gene control of AvrLm1, AvrLm2, AvrLm4, AvrLm5, and AvrLm8 (Table 6). In contrast, a 75:25 A/V segregation ratio was observed on Falcon-MX, suggesting that in this cross two genes controlled avirulence on Falcon-MX (Table 6). As in cross 20, all AvrLm4 isolates (i.e., avirulent on Jet Neuf) were also avirulent on Falcon-MX, but avrLm4 isolates (i.e., virulent on Jet Neuf) were 50:50 A/V on Falcon-MX (16A:18V; chi-square $=0.118 ; P=0.732)$. These data are consistent with the hypothesis that the digenic control of avirulence on Falcon-MX in cross 34 is due to the presence of both Rlm4 and Rlm6 in this line, as suggested from cross 20 segregation data. Consequently, the $50: 50 \mathrm{~A} / \mathrm{V}$ ratio of avrLm4 isolates on Falcon-MX indicates that AvrLm6 and AvrLm4 are unlinked. Because of the presence of Rlm4 in Falcon-MX, the genotype of AvrLm4 isolates at the AvrLm6 locus could not be inferred and, for these isolates, data at the AvrLm6 locus were considered missing data for linkage analysis. In cross 34 , five recombination events (out of 61) were detected between AvrLml and AvrLm2 (8.7 cM), whereas only one recombination event was observed between AvrLm6 and AvrLm1, and two between AvrLm6 and AvrLm2. Mapmaker analysis of cross 34 segregation data indicated that AvrLm6 mapped between AvrLm1 (2.9 cM) and AvrLm2 (5.8 cM). Linkage analysis from crosses 29 and 34 further indicated that, conversely to the cluster formed by AvrLm1, AvrLm2, and AvrLm6, the AVR loci AvrLm4, AvrLm5, and AvrLm8 were all independent from each other and from the AvrLml region.

Novel specific interactions toward B. napus: AvrLm3 specifies incompatibility between IBCN78 or IBCN79 and Glacier. Isolates IBCN78 and IBCN79 displayed a new specific interaction because they were avirulent on $\mathrm{cv}$. Glacier but virulent on $\mathrm{cv}$. Bristol (Table 3). In addition, these isolates were the only ones to induce a resistance response in B. napus line 22-1-1 (Table 3). We thus postulated the occurrence of a new interaction involving one AVR allele present in IBCN78 and IBCN79, termed AvrLm3, and one resistance gene, $R \operatorname{lm} 3$, present in Glacier and 22-1-1, but not in Bristol (Tables 7 and 8). To check this hypothesis, two crosses were analyzed. In cross 35 , isolate IBCN78 was crossed with v23.1.11 (AvrLm1-AvrLm4) (Tables 1 to 3 and 7). The entire progeny was virulent on Bristol, as expected under the hypothesis that none of the parental isolates possessed AvrLm2 (Table 7). The progeny was $100 \%$ avirulent on Doublol and Columbus, suggesting that IBCN78 also possessed AvrLml. A nonexclusive hypothesis, however, would be that Doublol and Columbus also possessed the putative $\mathrm{R}$ gene Rlm3, and that AvrLml and the putative

TABLE 6. Segregation analysis of avirulence of Leptosphaeria maculans toward resistance sources present in Brassica juncea and B. rapa

\begin{tabular}{|c|c|c|c|c|c|c|c|c|}
\hline & \multicolumn{8}{|c|}{ Brassica lines and known or putative resistance genes ${ }^{\mathrm{a}}$} \\
\hline & \multicolumn{4}{|c|}{ B. napus } & \multicolumn{3}{|c|}{ B. juncea } & \multirow[b]{2}{*}{ B. rapa } \\
\hline & & Bristol & Jet Neuf & Vivol Capitol & & $150-2-1$ & Falcon_MX & \\
\hline & Westar & $R \operatorname{lm} 2$ & $\operatorname{Rlm} 4$ & $(R \operatorname{lm} 3)$ & $(R \operatorname{lm} 5)(R \operatorname{lm} 6)$ & $(R \operatorname{lm} 5)$ & $(R \operatorname{lm} 4)(R \operatorname{lm} 6)$ & $156-2-1(\operatorname{Rlm} 8)$ \\
\hline \multicolumn{9}{|l|}{ Cross 24} \\
\hline \multicolumn{9}{|l|}{ Parental isolates } \\
\hline 22.2 .03 & $\mathrm{~V}^{\mathrm{b}}$ & $\mathrm{V}$ & $\mathrm{V}$ & A & A & $\mathrm{V}$ & A & A \\
\hline $\mathrm{v} 11.1 .5$ & $\mathrm{~V}$ & $\mathrm{~V}$ & $\mathrm{~V}$ & A & A & A & A & A \\
\hline Number of progeny tested & 33 & 20 & 0 & 56 & 28 & 43 & 0 & 0 \\
\hline $\mathrm{A} / \mathrm{V}$ isolates in the progeny ${ }^{\mathrm{c}}$ & $0: 33$ & $0: 20$ & $\mathrm{nd}^{\mathrm{d}}$ & $56: 0$ & 28:0 & $22: 21$ & nd & nd \\
\hline Chi-square $(50: 50)^{\mathrm{e}}$ & - & - & - & - & - & 0.023 & _- & - \\
\hline$P$ value & - & - & - & - & - & $0.879 \mathrm{NS}^{\mathrm{f}}$ & - & - \\
\hline \multicolumn{9}{|l|}{ Cross 29} \\
\hline \multicolumn{9}{|l|}{ Parental isolates } \\
\hline 22.2 .02 & $\mathrm{~V}$ & A & $\mathrm{V}$ & $\mathrm{V}$ & A & A & $\mathrm{V}$ & $\mathrm{V}$ \\
\hline 22.2 .03 & $\mathrm{~V}$ & $\mathrm{~V}$ & $\mathrm{~V}$ & A & A & $\mathrm{V}$ & A & A \\
\hline Number of progeny tested & 67 & 67 & 0 & 67 & 51 & 62 & 67 & 67 \\
\hline $\mathrm{A} / \mathrm{V}$ isolates in the progeny & $0: 67$ & $32: 35$ & nd & $36: 31$ & $45: 6$ & $37: 25$ & $35: 32$ & $30: 37$ \\
\hline Chi-square $(50: 50)$ & - & 0.134 & - & 0.373 & 29.8 & 2.323 & 0.134 & 0.731 \\
\hline$P$ value & _- & $0.714 \mathrm{NS}$ & - & $0.541 \mathrm{NS}$ & $<0.001 * * *$ & $0.128 \mathrm{NS}$ & $0.714 \mathrm{NS}$ & $0.392 \mathrm{NS}$ \\
\hline Chi-square $(75: 25)$ & - & 26.5 & - & 16.16 & 4.76 & 7.76 & 18.51 & 32.64 \\
\hline$P$ value & - & $<0.001 * * *$ & - & $<0.001 * * *$ & $0.029 *$ & $0.005^{* *}$ & $<0.001 * * *$ & $<0.001 * * *$ \\
\hline \multicolumn{9}{|l|}{ Cross 34} \\
\hline \multicolumn{9}{|l|}{ Parental isolates } \\
\hline v29.3.1 & $\mathrm{V}$ & A & $\mathrm{V}$ & $\mathrm{V}$ & $\mathrm{V}$ & $\mathrm{V}$ & $\mathrm{V}$ & $\mathrm{V}$ \\
\hline v23.1.3 & $\mathrm{V}$ & $\mathrm{V}$ & A & A & A & A & A & A \\
\hline Number of progeny tested & 61 & 61 & 61 & 61 & $7 \mathrm{~g}$ & 59 & 61 & 59 \\
\hline $\mathrm{A} / \mathrm{V}$ isolates in the progeny & $0: 61$ & $32: 29$ & $27: 34$ & $28: 33$ & $4: 3$ & $35: 24$ & $43: 18$ & $31: 28$ \\
\hline Chi-square $(50: 50)$ & - & 0.148 & 0.803 & 0.410 & 0.143 & 2.051 & 10.24 & 0.153 \\
\hline$P$ value & _- & $0.701 \mathrm{NS}$ & $0.370 \mathrm{NS}$ & $0.522 \mathrm{NS}$ & $0.705 \mathrm{NS}$ & $0.152 \mathrm{NS}$ & $0.001 *$ & $0.696 \mathrm{NS}$ \\
\hline Chi-square $(75: 25)$ & - & 16.54 & 30.74 & 27.55 & 1.19 & 7.73 & 0.661 & 15.87 \\
\hline$P$ value & - & $<0.001 * * *$ & $<0.001 * * *$ & $<0.001 * * *$ & $0.27 \mathrm{NS}$ & $0.005^{* *}$ & $0.416 \mathrm{NS}$ & $<0.001 * * *$ \\
\hline
\end{tabular}


AvrLm3 allele are linked enough to prevent the recovery of recombinant isolates within the limited number of progeny analyzed. The avirulent phenotype of IBCN78 on both Glacier and 22-1-1 was inherited with a ratio fitting the 50:50 expected ratio for a single gene control (Table 7). All avirulent progeny on Glacier were also avirulent on line 22-1-1. In addition, the avirulent phenotype of the parental isolate v23.1.11 on Jet Neuf was inherited as a single gene, as expected if only v23.1.11 possessed the AVR allele AvrLm4 (Table 7). Finally, no recombinant isolates, displaying either avirulence on Glacier, Jet Neuf, and 22-1-1 or virulence on these three lines, could be recovered, because only the two parental phenotypic classes were detected among the progeny (data not shown). All these data indicated that one AVR locus is involved in the genetic control of avirulence of IBCN78 on Glacier and 22-1-1, but that this locus is tightly linked (or identical) to the AvrLm4 locus. Because AvrLml and AvrLm4 are unlinked (7; this study), AvrLm1 and AvrLm3 must also be independent. Therefore, the lack of virulent isolates on Doublol and Columbus in cross 35 progeny strongly suggests that IBCN78 also possesses the AvrLml allele.

Cross 19 (IBCN79 $\times$ H5) progeny was recovered as tetrads. Only one complete tetrad and six incomplete tetrads (i.e., in which

TABLE 7. Segregation analysis of the differential interaction of the Leptosphaeria maculans isolate IBCN78 toward the Brassica napus cvs. Bristol and Glacier

\begin{tabular}{|c|c|c|c|c|c|}
\hline & \multicolumn{5}{|c|}{ Brassica lines and known or putative resistance genes ${ }^{\mathrm{a}}$} \\
\hline & $\begin{array}{l}\text { Westar (none) } \\
\text { Bristol Rlm2 }\end{array}$ & $\begin{array}{l}\text { Glacier } R \operatorname{lm} 2(R \operatorname{lm} 3) \\
22-1-1(R \operatorname{lm} 3)\end{array}$ & $\begin{array}{l}23-1-1 \\
(\operatorname{Rlm} 7)\end{array}$ & $\begin{array}{l}\text { Jet Neuf } \\
\text { Rlm4 }\end{array}$ & $\begin{array}{l}\text { Columbu, Doublol } \\
\quad \operatorname{Rlm} 1 \text { (Rlm } 3)\end{array}$ \\
\hline \multicolumn{6}{|l|}{ Cross 35-parental isolates } \\
\hline IBCN78 & $\mathrm{V}^{\mathrm{b}}$ & A & $\mathrm{V}$ & $\mathrm{V}$ & A \\
\hline $\mathrm{v} 23.1 .11$ & $\mathrm{~V}$ & $\mathrm{~V}$ & A & A & A \\
\hline Number of progeny tested & 39 & 39 & 39 & 39 & 39 \\
\hline $\mathrm{A} / \mathrm{V}$ isolates in the progeny ${ }^{\mathrm{c}}$ & $0: 39$ & $18: 21$ & $21: 18$ & $21: 18$ & 39:0 \\
\hline Chi-squared $^{d}$ & - & 0.231 & 0.231 & 0.231 & - \\
\hline$P$ value & - & $0.631 \mathrm{NS}^{\mathrm{e}}$ & $0.631 \mathrm{NS}$ & $0.631 \mathrm{NS}$ & - \\
\hline
\end{tabular}

${ }^{a} \mathrm{R}$ genes between brackets are putative $\mathrm{R}$ genes deduced from the present study.

${ }^{\mathrm{b}} \mathrm{A}$, the isolate is avirulent; $\mathrm{V}$, the isolate is virulent.

${ }^{c}$ The values given are number of isolates in each phenotypic class A (avirulent) or V (virulent).

$\mathrm{d}$ Chi-square value for the single gene control hypothesis (50:50 A/V ratio).

${ }^{\mathrm{e}} \mathrm{NS}$, the observed ratio is not significantly different from the tested hypothesis at a $5 \%$ level; -, the interaction phenotype on this line or cultivar is not segregating in this cross.

TABLE 8. Tetrad analysis of avirulence segregation of Leptosphaeria maculans progeny from cross 19 (IBCN79 $\times$ H5) on a range of Brassica napus lines

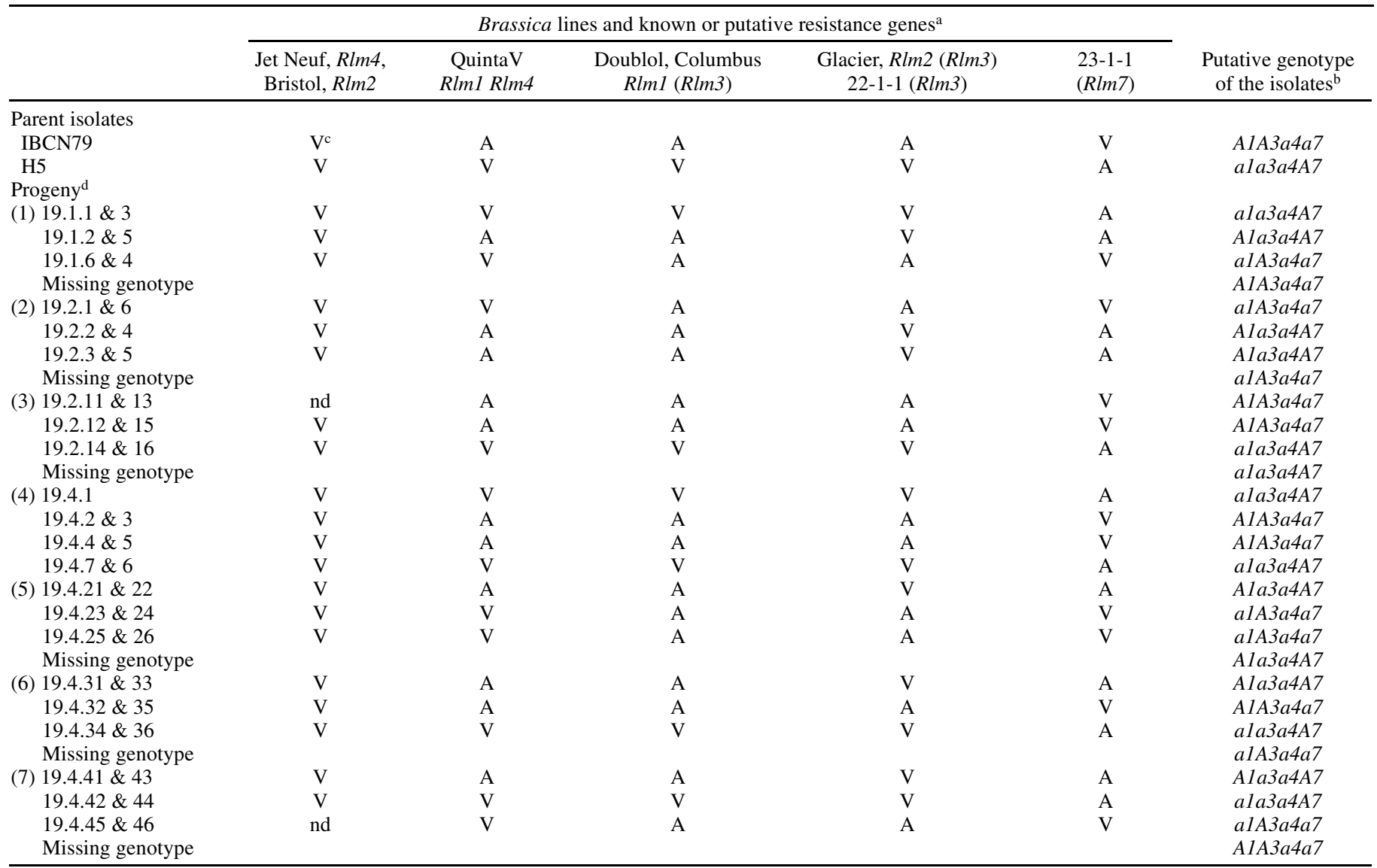

$\overline{\mathrm{R}}$ genes between brackets are putative $\mathrm{R}$ genes deduced from the present study.

${ }^{\mathrm{b}} \mathrm{Ai}$, presence of the avirulent allele at the AvrLmi locus; $a i$, presence of the virulent allele at the AvrLmi locus.

${ }^{\mathrm{c}} \mathrm{A}$, the isolate is avirulent; $\mathrm{V}$, the isolate is virulent; nd, not determined.

d Each tetrad is identified by its number, indicated between brackets. Isolates on the same line are twin isolates, as identified following pulsed field gel electrophoresis. 
one of the four products of meiosis was missing) were recovered (Table 8). The entire progeny was virulent on Jet Neuf and Bristol, which confirmed that both parents possess the virulent alleles avrLm2 and avrLm4 (Table 8). All interactions segregating in cross 19 segregated 2:2 in the complete tetrad (tetrad 4) (Table 8). Although incomplete, all other tetrads displayed segregation data that are consistent with a 2:2 A/V ratio on both QuintaV and the two putative $R \operatorname{lm} 3$ genotypes. In contrast, such a 2:2 segregation was not observed on Columbus and Doublol, in at least two of the six incomplete tetrads (i.e., tetrads 2 and 5) (Table 8), because the three recovered genotypes of each of these two tetrads were all avirulent on these lines. In addition, recombinant isolates, i.e., virulent on QuintaV but avirulent on Columbus and Doublol, were recovered in tetrads $1,2,5$, and 7 (Table 8). Finally, all progeny isolates from cross 19 that were avirulent on either QuintaV or on the two putative Rlm3 lines were also avirulent on Columbus. All these data support the hypothesis that AvrLm3 is present in IBCN79 and matches Rlm3 present in 22-1-1 and Glacier, but also that (i) AvrLml is present in IBCN79 and (ii) $R \operatorname{lm} 3$ is present in Columbus and Doublol, but not in QuintaV. Under these hypotheses, the recovery of the recombinant genotypes AvrLm1-avrLm3 and avrLm1-AvrLm3 in four of seven tetrads confirms the genetic independence between AvrLm1 and AvrLm3.

Novel specific interactions toward $B$. napus: virulence of isolates $\mathrm{Nz}-\mathrm{T4}$, IBCN78, and IBCN79 on $B$. napus resistance sources is under a single gene control. Screening of $B$. napus gene banks for resistance to European PG4 isolates, i.e., having virulent alleles at the AvrLm1, AvrLm2, and AvrLm4 loci, allowed us to select five plant genotypes, from diverse geographic origin, that displayed an identical interaction phenotype, i.e., resistance to PG4 isolates and susceptibility to Nz-T or its single-conidia progeny Nz-T4 (lines 149-2-1, 148-1-1, 05-1-1, 04-1-2, and 23-1-1) (Table 3). Most of these lines were also susceptible to IBCN78 and IBCN79 (Table 3), suggesting both that avirulence on these resistance sources may be under a simple genetic control and that all these lines may possess the same $\mathrm{R}$ gene. The progeny of cross $30(\mathrm{Nz}-\mathrm{T} 4 \times \mathrm{v} 23.1 .2)$ was analyzed for avirulence on selected genotypes 148-1-1, 149-2-1, and 23-1-1. All progeny analyzed displayed the same interaction phenotype on each of these lines, with an $\mathrm{A} / \mathrm{V}$ ratio fitting the 50:50 ratio expected for a single gene control of avirulence (Table 9). It was thus postulated that one single locus governs avirulence on these new resistant lines. The corresponding putative gene pair was termed AvrLm7/Rlm7. To further analyze the genetic linkage between this locus and AvrLm1 and AvrLm4, cross 33 was performed between isolate Nz-T4 and the AvrLm1-AvrLm4 isolate, v23.1.11 (Tables 1 to 3). Avirulence toward Jet Neuf, Columbus, and the B. napus lines 148-1-1 and 23-1-1 all segregated in cross 33 as monogenic traits (Table 9). Four phenotypic classes were observed among cross 33 progeny, i.e., the two parental phenotypic classes, and two phenotypic classes showing recombination between avirulence toward Columbus (due to the interaction between AvrLm1 present in isolate v23.1.11 and Rlml in Columbus) and avirulence toward both Jet Neuf and lines 148-1-1 and 23-1-1 (Table 10). These four phenotypic classes were recovered in proportions fitting the 25:25:25:25 ratio (chi-square $=2.17 ; \mathrm{df}=3 ; P=0.538 \mathrm{NS}$ ). No recombinant isolates were recovered between avirulence toward Jet Neuf, and virulence on 148-1-1 and 23-1-1 (Table 10). Segregation data from cross 33 thus confirmed the single gene control of avirulence on lines 148-1-1 and 23-1-1, but also indicated that the corresponding locus is independent from $A v r L m 1$, but tightly linked (or allelic) to AvrLm4.

In independent crosses 19 and 35, the parental isolates IBCN78 and IBCN79 displayed virulence on resistant lines 23-1-1, 148-11 , and 149-2-1 (Table 3). As in cross 33, specific interaction on 23-1-1 was under a single gene control (Tables 7 and 8; data not shown). In cross 35 , the corresponding locus also appeared to be tightly linked, or identical, to the AvrLm4 locus because no recombinant isolates could be recovered in the progeny. Avirulence on line 23-1-1 also segregated in cross 19 (Table 8) with a 2:2 ratio in the complete tetrad, and with a $1: 2$ or $2: 1$ ratio in incomplete tetrads, i.e., still fitting a single gene control. In cross 19, AvrLm7 was tightly linked, or identical, to the AvrLm3 locus (Table 8).

\section{DISCUSSION}

In this paper, we report on the genetic identification of five novel avirulence genes or alleles: (i) AvrLm3, specifying incompatibility on cv. Glacier but genetically independent from AvrLm2; (ii) two independent loci, AvrLm5 and AvrLm6, inducing resistance on B. juncea; (iii) AvrLm7, specifying incompatibility on a novel B. napus resistance source to PG4-A1 (i.e., avrLm1-

TABLE 9. Segregation analysis of the differential interaction of the Leptosphaeria maculans isolate Nz-T4 toward a range of Brassica napus cultivars or lines

\begin{tabular}{|c|c|c|c|c|c|c|}
\hline & \multicolumn{6}{|c|}{ Brassica lines and known or putative resistance genes ${ }^{\mathrm{a}}$} \\
\hline & Westar & $\begin{array}{c}\text { Bristol } \\
\text { Rlm2 }\end{array}$ & $\begin{array}{c}149-2-1 \\
(R \operatorname{lm} 1)(R \operatorname{lm} 7)\end{array}$ & $\begin{array}{c}148-1-1,23-1-1 \\
(R \operatorname{lm} 7)\end{array}$ & $\begin{array}{l}\text { Jet Neuf } \\
\text { Rlm4 }\end{array}$ & $\begin{array}{l}\text { Columbus } \\
R \operatorname{lm} 1(R \operatorname{lm} 3)\end{array}$ \\
\hline \multicolumn{7}{|l|}{ Cross 30 parental isolates } \\
\hline $\mathrm{Nz}-\mathrm{T} 4$ & $\mathrm{~V}^{\mathrm{b}}$ & $\mathrm{V}$ & $\mathrm{V}$ & V & $\mathrm{V}$ & V \\
\hline $\mathrm{v} 23.1 .2$ & $\mathrm{~V}$ & $\mathrm{~V}$ & A & A & $\mathrm{V}$ & $\mathrm{V}$ \\
\hline Number of progeny tested & 66 & $\mathrm{nd}^{\mathrm{c}}$ & 66 & 64 & nd & nd \\
\hline $\mathrm{A} / \mathrm{V}$ isolates in the progeny ${ }^{\mathrm{d}}$ & $0: 66$ & - & $36: 30$ & $34: 30$ & - & - \\
\hline Chi-square value $(50: 50 \text { ratio })^{\mathrm{e}}$ & - & - & 0.545 & 0.250 & - & - \\
\hline$P$ value & - & - & $0.46 \mathrm{NS}^{\mathrm{f}}$ & $0.617 \mathrm{NS}$ & - & - \\
\hline \multicolumn{7}{|l|}{ Cross 33 parental isolates } \\
\hline $\mathrm{Nz}-\mathrm{T} 4$ & $\mathrm{~V}$ & $\mathrm{~V}$ & $\mathrm{~V}$ & $\mathrm{~V}$ & $\mathrm{~V}$ & $\mathrm{~V}$ \\
\hline v23.1.11 & $\mathrm{V}$ & $\mathrm{V}$ & A & A & A & A \\
\hline Number of progeny tested & 32 & 33 & nd & 48 & 48 & 48 \\
\hline $\mathrm{A} / \mathrm{V}$ isolates in the progeny & $0: 32$ & $0: 33$ & - & $27: 21$ & $27: 21$ & $28: 20$ \\
\hline Chi-square value (50:50 ratio) & - & - & - & 0.75 & 0.75 & 1.333 \\
\hline$P$ value & - & - & - & $0.386 \mathrm{NS}$ & $0.386 \mathrm{NS}$ & $0.248 \mathrm{NS}$ \\
\hline Chi-square value (75:25 ratio) & - & - & - & 8.999 & 8.999 & 7.111 \\
\hline$P$ value & - & - & - & $0.0027 * *$ & $0.0027 * *$ & $0.007 * *$ \\
\hline
\end{tabular}

a $\mathrm{R}$ genes between brackets are putative $\mathrm{R}$ genes deduced from the present study.

b A, the isolate is avirulent; $\mathrm{V}$, the isolate is virulent.

$\mathrm{c}$ nd, segregation analyses not performed on this Brassica line.

$\mathrm{d}$ The values given are number of isolates in each phenotypic class (A or V).

e The expected A/V ratio for the tested hypothesis is indicated between brackets (50:50, single gene control; 75:25, digenic control with two unlinked genes).

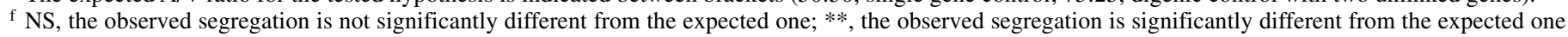
(1\% level). 
avrLm2-avrLm4) isolates; and (iv) AvrLm8, recognizing a resistance source to PG4-A1 isolates present in B. rapa.

Although numerous interactions were described between $L$. maculans and B. napus cultivars or lines $(9,19,20,23,24)$, only a few of them were subjected to genetic analyses $(1,2,7,16)$. One main difficulty that probably slowed down such analyses was the frequent occurrence of numerous AVR alleles in field L. maculans isolates and numerous $\mathrm{R}$ genes, with epistatic action, in B. napus cultivars. The presence of both Rlml and Rlm4 in some, but not all, B. napus cv. Quinta-derived lines was reported previously (7). Similarly, it is suggested from the present study that numerous commercial cultivars possess both $R \operatorname{lm} 1$ and $R \operatorname{lm} 3$, whereas the differential cv. Glacier possesses Rlm 2 and $R \operatorname{lm} 3$. On the fungal side, most of the field isolates subjected to genetic analyses possess more than one AVR allele. For instance, PHW1245 possesses at least AvrLm1, AvrLm2, AvrLm4, and AvrLm5-AvrLm8. The four AVR alleles AvrLm1, AvrLm2, AvrLm4, and AvrLm7 were identified in isolate IBCN18. Similarly, European PG4 isolates typically possess four AVR alleles (AvrLm5 to AvrLm8). One of the main deliverables of the present study is the obtainment, following in vitro crosses, of isolates with a limited number of AVR alleles. This was the case for isolate 22.2.02, which possesses only the AVR alleles AvrLm2, AvrLm5, and AvrLm7. To our knowledge, this is the first record of genetic characterization of one isolate being avirulent on cv. Glacier (and Bristol and other cultivars possessing $R \operatorname{lm} 2$ ) but virulent on cv. Quinta, which constitutes a very useful tool to identify the presence of Rlm2 in B. napus cultivars or segregating populations. Similarly, we genetically characterized a few avrLm1-avrlm2-AvrLm3-avrlm4 isolates, in progeny of cross 19, which will help us identify the presence of Rlm3 in B. napus lines, and which will be used to map this gene. However, additional in vitro crosses are still needed to obtain $L$. maculans isolates possessing only one AVR allele for all AVR loci described here, in order to further improve the L. maculans differential set.

Five $B$. napus lines were independently selected as new sources of resistance to European PG4-A1 isolates. Three isolates from diverse geographic origin (Nz-T, IBCN78, and IBCN79) were however virulent on these lines. Genetic studies demonstrated that (i) the virulence of all three isolates on these lines was under a simple genetic control; and (ii) the corresponding loci mapped identically in the three isolates (i.e., cosegregating with AvrLm4 and/or AvrLm3). Although allelic tests were not performed between these three isolates, it can thus be hypothesized that Nz-T, IBCN78, and IBCN79 all possess the same virulence allele, termed $\operatorname{avr} \operatorname{Lm} 7$, interacting with the putative $\mathrm{R}$ gene, $\operatorname{R} \operatorname{lm} 7$, present in all these lines. These results support the assumption that the Nz-T isolate belongs to a new race of $L$. maculans, as proposed by Gowers and Armstrong (19).

Although differential interactions between $B$. rapa lines and $L$. maculans isolates were described previously $(6,22-24)$, the genetic control of these interactions was not elucidated. Mainly, screening of worldwide collections of $B$. rapa for resistance to PG3 and PG4 isolates indicated a continuous variation in $L$. maculans resistance, with no plant genotypes being completely susceptible or completely resistant to either pathotypes (27). However, the same authors demonstrated that after four generations of selection for resistance to a PG4 isolate, using the cotyledon inoculation test, the mean disease severity shifted from 8.11 to 2.42, on a 0 -to-9 scale, with a clear bimodal distribution of IC after the third selection generation (27). These results clearly indicate that major resistance genes to $L$. maculans could be present, although at low frequencies, in $B$. rapa lines. The $B$. rapa line 156-2-1 described in the present study was obtained similarly, because 1 resistant plant out of 12 was initially selected from a $B$. rapa commercial cultivar. After two selfing/selection generations, the resulting line contained more than $95 \%$ of resistant plants (data not shown). The genetic control of avirulence to B. rapa was clearly monogenic and the corresponding locus, termed AvrLm8, was genetically unlinked to any other $L$. maculans AVR gene.

To date, the L. maculans-B. juncea interaction was mainly genetically analyzed on the plant side, and depending on the authors, a single, digenic, or trigenic control was reported $(13,21,31,37)$. The occurrence of two independent $\mathrm{R}$ genes in $B$. juncea was supported by genetic analysis of resistance to $L$. maculans either from segregating populations, including $\mathrm{DH}$ lines, or from addition or recombination lines $(12,21,31)$. Analysis of F1, F2, and F3 from three independent crosses between resistant and susceptible $B$. juncea accessions demonstrated the occurrence of two nuclear genes, one dominant and one recessive with epistatic action, that control the B. juncea resistance to L. maculans (21). It was thus expected to find at least two AVR genes from the pathogen, each one recognizing one of these $\mathrm{R}$ genes, especially when the parent isolate used was virulent on many unrelated $B$. juncea cultivars, such as Picra, Aurea (this study), Stoke, and Zaria (11) along with 25 additional $B$. juncea accessions from diverse geographic origin (30). The genetic analyses presented here indicate that the $L$. maculans $-B$. juncea interaction is controlled by two pairs of AVR/R genes, i.e., AvrLm5/Rlm5 and AvrLm6/Rlm6, with epistatic action between the two $\mathrm{R}$ genes. Our results suggest that AvrLm6 interacts with the B. juncea locus Jlm1 (termed Rlm6 in the present study), introgressed into the Falcon-MX B. napus line (12). In contrast, we cannot yet establish whether AvrLm5 interacts with the resistance source described in $B$. nigra addition lines and located on chromosome B4 $(12,13)$ or with another R gene from $B$. juncea.

The virulence of isolate IBCN18 on B. juncea was previously genetically analyzed and a single gene control of virulence was described $(11,14)$. Our results therefore could appear to contradict those from Chen et al. (11), because we found three independent virulent alleles in IBNC18, two of them at least interacting with $\mathrm{R}$ genes present in $B$. juncea cultivars. Two hypotheses could however explain this apparent discrepancy. The first one would be that only one of the two putative B. juncea $\mathrm{R}$ genes, $R \operatorname{lm} 5$ or $R \operatorname{lm} 6$, was present in the $B$. juncea cv. Stoke used by Chen et al. (11) to characterize their progeny. In the present study, we obtained progeny isolates possessing either the virulent or the avirulent allele for each locus AvrLm5 and AvrLm6, which can now be used to test this hypothesis. The second hypothesis would be that the three alleles AvrLm5, AvrLm6, and AvrLm8 were not all segregating in the IBCN17 $\times$ IBCN18 cross studied by Chen et al. (11). We demonstrated that AvrLm5 and AvrLm8 were indeed segregating in the IBCN17 $\times$ IBCN18 cross, as revealed by the incompatible interaction obtained between IBCN17 and lines 150-2-1, 151-2-1, and 156-2-1 (data not shown). However, we could not conclude this as regards AvrLm6, because isolate IBCN17 was avirulent on Falcon-MX, but also on Jet Neuf and Falcon (data not shown),

TABLE 10. Interaction phenotypes of Leptosphaeria maculans progeny isolates from cross $33(\mathrm{Nz}-\mathrm{T} 4 \times \mathrm{v} 23.1 .11)$ on a range of Brassica napus cultivars or lines

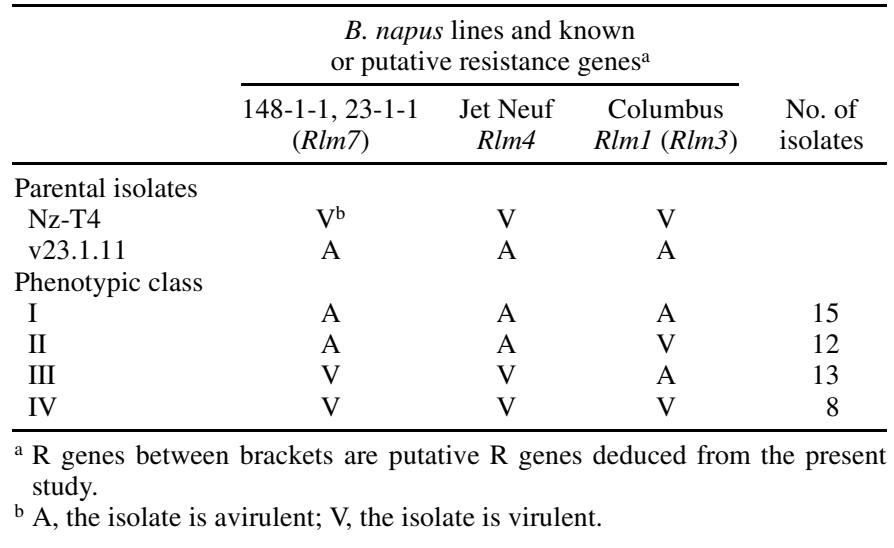

Vol. 92, No. 10, 2002 
indicating that IBCN17 possesses AvrLm4. This prevented us from characterizing IBCN17 genotype at the AvrLm6 locus due to the presence of both Rlm4 and Rlm6 in Falcon-MX (Table 4). If IBCN17 actually possesses the avrLm6 allele, then only AvrLm5 (and AvrLm8) segregates in the cross-analyzed by Chen et al. (11), and consequently the virulence locus mapped by Cozjinsen et al. (14) probably corresponds to AvrLm5. This is further supported by the physical mapping of this virulence locus on a chromosome whose size ranged from 1.80 to $1.85 \mathrm{Mb}$ (14), whereas AvrLm6 was found in the present study to be closely linked to AvrLm1, which is located on a chromosome whose size ranged from 2.48 to $3.10 \mathrm{Mb}$, depending on the isolate (3).

Linkage between AvrLm1 and AvrLm2 (2) was confirmed in the present study, and the genetic distance between the two loci was estimated to range from $1.5 \mathrm{cM}$ (cross 29) to $8.7 \mathrm{cM}$ (cross 34). Linkage analyzes demonstrated that AvrLm6 is tightly linked to, but not an allele of, AvrLm1 and AvrLm2, and is localized between AvrLm1 and AvrLm2. The AvrLm1 region has thus to be considered as a cluster of AVR genes, with three AVR genes in less than $10 \mathrm{cM}$. Importantly, the AvrLm1-AvrLm2-AvrLm6 cluster contains AVR genes corresponding to $\mathrm{R}$ genes originating not only from the $B$. napus species, but also from the related species $B$. juncea. AVR gene clusters have been described for only a few phytopathogenic fungi, such as Magnaporthe grisea, Phytophthora infestans, and P. sojae $(15,33,35)$, but clusters of AVR genes are reported in L. maculans for the first time. An identical situation may also exist at the AvrLm4 locus because we were not able, in the present study, to establish whether AvrLm3, AvrLm4, and AvrLm7 were three different allelic forms of one single locus or whether they are truly distinct, but clustered, AVR genes. The lack of recombination between AvrLm3, AvrLm4, and AvrLm7 has to be interpreted carefully, because for all crosses performed to analyze linkage between them, a relatively small number of progeny isolates was analyzed. In addition, crosses 19, 30, 33, and 35 in which AvrLm3, AvrLm4, and/or AvrLm7 were segregating, were all F1 crosses. In contrast, crosses 22, 24, and 29, for which recombination between loci AvrLm1, AvrLm2, and AvrLm6 was observed, were BC1 or F2. Due to the well-known chromosome size polymorphism in $L$. maculans $(26,28)$, it can be hypothesized that in F1 progeny from crosses between very genetically distant isolates (such as crosses 19, 30, 33, and 35 which all involved two isolates from different continents), the genome organization of the two parental isolates is so different that recombination cannot occur as frequently as in more inbred isolates (5). Additional BC are underway to further analyze the linkage between the putative genes AvrLm3, Avrlm4, and AvrLm7.

Finally, linkage analyses demonstrated that at least four independent regions of the L. maculans genome, i.e., the AvrLm1AvrLm2-AvrLm6 cluster, the AvrLm3-AvrLm4-AvrLm7 region, $A v r L m 5$, and AvrLm8, are involved in host specificity toward $B$. napus, B. rapa, or B. juncea. Molecular mapping of these AVR genes using the segregating populations described in the present study is in progress and will allow us to identify, among the 18 linkage groups identified to date in the L. maculans maps (5), which ones are bearing genes involved in host specificity.

\section{ACKNOWLEDGMENTS}

This work was supported by EU contract FAIR3CT96-1669, grants from the "Société interprofessionnelle des oléagineux, protéagineux et cultures textiles" (SIDO), the "Agence de l'environnement et de la maîtrise de l'énergie" (ADEME) and the French Ministry of Agriculture. We thank I. Lepneuveu, B. Letarnec, L. Coudard, and J. P. Janiault (INRA PMDV, Versailles, France) for plant management; S. Gowers (CFR, Christchurch, New Zealand) for the New Zealand isolate; B. Howlett (Melbourne University, Australia) for isolate IBCN18; P. H. Williams for isolates IBCN78 and IBCN79; B. Fitt (IACR, Rothamsted, UK) and J. S. Dias (ISA, Lisbon, Portugal) for the European isolates UK1 and PT1; E. Willner (IPK Gatersleben, Germany) and F. N. Green
(SASA, Edinburgh, UK) for the collections of Brassica genotypes; M. Renard (INRA Le Rheu, France) for the Falcon-MX line; and J. West (IACR, Rothamsted, UK) for review of the manuscript.

\section{LITERATURE CITED}

1. Ansan-Melayah, D., Balesdent, M.-H., Buée, M., and Rouxel, T. 1995. Genetic characterization of AvrLml, the first avirulence gene of Leptosphaeria maculans. Phytopathology 85:1525-1529.

2. Ansan-Melayah, D., Balesdent, M.-H., Delourme, R., Pilet, M. L., Tanguy, X., Renard, M., and Rouxel, T. 1998. Genes for race-specific resistance against blackleg disease in Brassica napus L. Plant Breed. 117:373-378.

3. Attard, A. 2001. Le gène d'avirulence AvrLm1 chez l'ascomycète phytopathogène Leptosphaeria maculans: Marquage moléculaire et caractérisation de son environnement génomique. Ph.D. thesis. Institut National Agronomique Paris-Grignon, Paris, France.

4. Attard, A., Gourgues, M., Gout, L., Schmit, J., Roux, J., Narcy, J. P., Balesdent, M. H., and Rouxel, T. 2001. Molecular characterisation and polymorphism of MinLm1, a minisatelite from the phytopathogenic ascomycete Leptosphaeria maculans. Curr. Genet. 40:54-64.

5. Attard, A., Gout, L., Gourgues, M., Khün, M. L., Schmit, J., Laroche, S., Ansan-Melayah, D., Billault, A., Cattolico, L., Balesdent, M. H., and Rouxel, T. 2002. Analysis of molecular markers genetically linked to the Leptosphaeria maculans avirulence gene AvrLml in field populations indicates a highly conserved event leading to virulence on RIml genotypes. Mol. Plant-Microbe Interact. 15:672-682.

6. Badawy, H. M. A., Hoppe, H. H., and Koch, E. 1991. Differential reactions between the genus Brassica and aggressive single spore isolates of Leptosphaeria maculans. J. Phytopathol. 131:109-119.

7. Balesdent, M. H., Attard, A., Ansan-Melayah, D., Delourme, R., Renard, M., and Rouxel, T. 2001. Genetic control and host range of avirulence toward Brassica napus cultivars Quinta and Jet Neuf in Leptosphaeria maculans. Phytopathology 91:70-76.

8. Balesdent, M.-H., Gall, C., Robin, P., and Rouxel, T. 1992. Intraspecific variation in soluble mycelial protein and esterase patterns of Leptosphaeria maculans French isolates. Mycol. Res. 96:677-684.

9. Ballinger, D. J., Salisbury, P. A., and Kadkol, G. P. 1991. Race variation in Leptosphaeria maculans and its implications for resistance breeding in Australia. Pages 226-231 in: Proc. Int. Rapeseed Congr., 8th.

10. Chen, C. Y., and Howlett, B. J. 1996. Rapid necrosis of guard cells is associated with the arrest of fungal growth in leaves of Indian mustard (Brassica juncea) inoculated with avirulent isolates of Leptosphaeria maculans. Physiol. Mol. Plant Pathol. 48:73-81.

11. Chen, C. Y., Plummer, K. M., and Howlett, B. J. 1996. Ability of a Leptosphaeria maculans isolate to form stem canker on Indian mustard (Brassica juncea) segregates as a single locus. Eur. J. Plant Pathol. 102:349-352.

12. Chèvre, A. M., Barret, P., Eber, F., Dupuy, P., Brun, H., Tanguy, X., and Renard, M. 1997. Selection of stable Brassica napus-B. juncea recombinant lines resistant to blackleg (Leptosphaeria maculans). 1. Identification of molecular markers, chromosomal and genomic origin of the introgression. Theor. Appl. Genet. 95:1104-1111.

13. Chèvre, A. M., Eber, F., This, P., Barret, P., Tanguy, X., Brun, H., Delseny, M., and Renard, M. 1996. Characterization of Brassica nigra chromosomes and of blackleg resistance in B. napus-B. nigra addition lines. Plant Breed. 115:113-118.

14. Cozjinsen, A. J., Popa, K. M., Roll, B. D., Purwantara, A., and Howlett, B. J. 2000. Genome analysis of the plant pathogenic fungus Leptosphaeria maculans: Mapping mating type and host specificity locus. Mol. Plant Pathol. 1:293-302.

15. Dioh, W., Tharreau, D., Notteghem, J. L., Orbach, M., and Lebrun, M. H. 2000. Mapping of avirulence genes in the rice blast fungus, Magnaporthe grisea, with RFLP and RAPD markers. Mol. Plant-Microbe Interact. 13:217-227.

16. Ferreira, M. E., Rimmer, S. R., Williams, P. H., and Osborn, T. C. 1995. Mapping loci controlling Brassica napus resistance to Leptosphaeria maculans under different screening conditions. Phytopathology 85:213217.

17. Gall, C., Balesdent, M. H., Desthieux, I., Robin, P., and Rouxel, T. 1995. Polymorphism of Tox $^{0}$ Leptosphaeria maculans isolates as revealed by soluble protein and isozyme electrophoresis. Mycol. Res. 99:221-229.

18. Gall, C., Balesdent, M. H., Robin, P., and Rouxel, T. 1994. Tetrad analysis of acid phosphatase, soluble protein patterns, and mating type in Leptosphaeria maculans. Phytopathology 84:1299-1305.

19. Gowers, S., and Armstrong, S. D. 1997. Breeding and selection for dry rot (Leptosphaeria maculans) resistance in Swedes (Brassica napus spp. rapifera). Page 52 in: ISHS Symposium on Brassicas. Tenth Crucifer Genetics Workshop, Rennes, France. 
20. Keri, M. 1999. Genetic studies of host-pathogen interaction between Brassica napus and Leptosphaeria maculans. Ph.D. thesis. University of Manitoba Winnipeg, Manitoba, Canada.

21. Keri, M., Van den Berg, C. G. J., Mc Vetty, P. B. E., and Rimmer, S. R. 1997. Inheritance of resistance to Leptosphaeria maculans in Brassica juncea. Phytopathology 87:594-598.

22. Koch, E., Song, K., Osborn, T. C., and Williams, P. H. 1991. Relationship between pathogenicity and phylogeny based on restriction fragment length polymorphism in Leptosphaeria maculans. Mol. Plant-Microbe Interact. 4:341-349.

23. Kuswinanti, T., Sock, J., and Hoppe, H. H. 1995. Variation in virulence of aggressive isolates of Leptosphaeria maculans based on cotyledon reactions on an extended differential set. Pages 1248-1250 in: Proc. Int. Rapeseed Congr., 9th. Cambridge, U.K.

24. Kutcher, H. R., van den Berg, C. G. J., and Rimmer, S. R. 1993. Variation in pathogenicity of Leptosphaeria maculans on Brassica spp. based on cotyledon and stem reactions. Can. J. Plant Pathol. 15:253-258.

25. Lammerink, J., and Hart, R. W. 1985. 'Tina', a new swede cultivar with resistance to dry rot and clubroot. N.Z. J. Exp. Agric. 13:417-420.

26. Leclair, S., Ansan-Melayah, D., Rouxel, T., and Balesdent, M. H. 1996. Meiotic behaviour of the minichromosome in the phytopathogenic ascomycete Leptosphaeria maculans. Curr. Genet. 30:541-548.

27. Mitchell-Olds, T., James, R. V., Palmer, M. J., and Williams, P. H. 1995. Genetics of Brassica rapa (syn. campestris). 2. Multiple disease resistance to three fungal pathogens: Peronospora parasitica, Albugo candida and Leptosphaeria maculans. Heredity 75:362-369.

28. Plummer, K. M., and Howlett, B. J. 1993. Major chromosomal length polymorphisms are evident after meiosis in the phytopathogenic fungus Leptosphaeria maculans. Curr. Genet. 24:107-113.

29. Pongam, P., Osborn, T. C., and Williams, P. H. 1998. Genetic analysis and identification of amplified fragment length polymorphism markers linked to the alm1 avirulence gene of Leptosphaeria maculans. Phytopathology 88:1068-1072.

30. Purwantara, A., Salisbury, P. A., Burton, W. A., and Howlett, B. J. 1998. Reaction of Brassica juncea (Indian mustard) lines to Australian isolates of Leptosphaeria maculans under glasshouse and field conditions. Eur. J. Plant Pathol. 104:895-902.

31. Rimmer, S. R., and Van den Berg, C. G. J. 1992. Resistance of oilseed Brassica spp. to blackleg caused by Leptosphaeria maculans. Can. J. Plant Pathol. 14:56-66.

32. Rouxel, T., and Séguin-Swartz, G. 1995. International blackleg of crucifers network. Blackleg News 5:15-16.

33. van der Lee, T., Testa, A., van't Klooster, J., van den Berg-Velthuis, G., and Govers, F. 2001. Chromosomal deletion in isolates of Phytophthora infestans correlates with virulence on $R 3, R 10$, and $R 11$ potato lines. Mol. Plant-Microbe Interact. 14:1444-1452.

34. Volke, B. 1999. Leptosphaeria maculans, der Erreger der Wurzelhalsund Stengelfäule an Raps: Verbreitung verschiedener Pathogenitätsgruppen in Europa, Quantifizierung des Befalls und Schadwirkung im Freiland. Ph.D. thesis. University of Göttingen, Germany.

35. Whisson, S. C., Drenth, A., Maclean, D. J., and Irwin, J. A. G. 1995. Phytophthora sojae avirulence genes, RAPD, and RFLP markers used to construct a detailed genetic linkage map. Mol. Plant-Microbe Interact. 8:988-995.

36. Williams, P. H., and Delwiche, P. A. 1979. Screening for resistance to blackleg of crucifers in the seedling stage. Pages 164-170 in: Proc. Eucarpia Conf. Breed. Cruciferous Crops. Wageningen, The Netherlands.

37. Zhu, J. S., Struss, D., and Robbelen, G. 1993. Studies on resistance to Phoma lingam in Brassica napus-Brassica nigra addition lines. Plant Breed. 111:192-197. 